\title{
POLAR WANDERING AND THE EARTH'S \\ DYNAMICAL EVOLUTION CYCLE
}

\author{
CHEH PAN \\ Westinghouse Georesearch Laboratory, Boulder, Colo., U.S.A.
}

\begin{abstract}
With a triaxial deformable Earth model an attempt is made to integrate the dynamics of polar wandering with that of the tectonic movements of the Earth's upper layers. Polar wandering is interpreted as a mechanical response of the rotating Earth to the episodic energy perturbation in the upper layers, whereas the tectonic movements are the adjustments of the upper layers to the polar instability. The present analysis suggests that the attempt of the Earth to adjust itself for polar stability is the driving mechanisms of continental drift and sea-fioor spreading, and also that the Earth's wobble is rather a quasi-permanent (slow damping) phenomenon and has two components. And that the Earth would not reach its rotational equilibrium as long as the excess heat-generation in its interior continues to supply energy for its cyclic dynamical evolution.
\end{abstract}

\section{Introduction}

The study of polar wandering has a long history, dated back to Sir George Darwin's work in 1877 (Munk, 1956; Munk and MacDonald, 1960). However, the progress of the study was slow; not until rather recently was a convincing qualitative discussion on this problem given by Gold (1955). The analytical treatments of Burgers (1955) and Inglis (1957) confirmed Gold's conclusion. Munk and MacDonald (1960, Ch. 12), on the other hand, made a thorough analytical study and full discussion of the problem but their conclusion was, 'the problem is unsolved'. Whereas Mansinha and Smylie (1967) attempted to interpret the secular polar motion as well as the Chandler wobble as an effect of earthquakes; the validity of the proposal was questioned by recent studies (Shimazaki and Takeuchi, 1972; Chinnery and Wells, 1972; Pan, 1971b).

Besides some palaeontological and palaeoclimatic evidence long known to geologists (Ma, 1960; Munk and MacDonald, 1960, p. 259-261), the observational evidence of polar wandering was rather scant until the discovery from paleomagnetic studies of the large-degree shift of the magnetic dipole during geological time. The recent astronomical observations (Markowitz, 1968; Yumi and Wako, 1968; Stoyko, 1968), however, show that at the present time the secular motion of the mean pole is about $0.003^{\prime \prime} / \mathrm{yr}$ or $10 \mathrm{~cm} / \mathrm{yr}$. This rate is somewhat greater than the rate of continental drift and sea-floor spreading, which ranges from about $1 \mathrm{~cm}$ to a few $\mathrm{cm}$ a year. However, the difference is insignificant and the two rates are of the same order of magnitude.

The confirmation of continental drift and sea-floor spreading from oceanographic and seismological observations has drastically revolutionized many conventional concepts in earth sciences. Nevertheless, the present studies of global tectonics are primarily observational and kinematical, and little effort has been made on the dynamics of the problem, particularly the dynamical relations between the tectonic movements and polar wandering. 
Gold (1955) first pointed out the instability of the Earth's rotation axis owing to the redistribution of mass in the Earth's interior. Pan (1963, 1968, 1970) discussed the relations between the energy-generation in the interior and the episodic nature of the Earth's evolution, and proposed a dynamical approach to the interpretation of the Earth's history. The present paper is a continuation of the previous work, or a further attempt to unify the dynamics of polar wandering, continental drift and sea-floor spreading. However, a full discussion and detailed mathematical analysis of the problem will be published elsewhere; this paper is a summary of the subject.

\section{The Triaxial Earth}

The Earth's triaxiality was first reported by Helmert (Munk and MacDonald, 1960, p. 163). But confirmation of triaxiality was not obtained until the appearance of satellite geodesy. Goldreich and Toomre (1969) demonstrated that the spherical harmonic coefficients of second degree of the geopotential obtained from satellite orbit perturbation indicate that the Earth is a triaxial body or a near-symmetric body of revolution. The difference between the two equatorial moments of inertia at the present time is about $0.002 \cdot 10^{42} \mathrm{~g} \mathrm{~cm}^{2}$, more than 130 times smaller than the difference between the longitudinal and equatorial moments of inertia. But the Earth's present moments of inertia are instantaneous and are not the principal moments of inertia of an equilibrium Earth; otherwise there would be no secular polar motion at the present time.

The Earth's equatorial ellipticity may be due largely to the mass and energy inhomogeneities in the interior (Goldreich and Toomre, 1969; Kaula, 1970; Pan, 1968, 1970). The Earth's upper layers are inhomogeneous; the fractured and multilayered lithosphere shows time-dependent strength whereas the weak asthenosphere yields viscously, depending upon the energy budget in the interior. As the rotation axis deviates from the axis of maximum principal moment of inertia, the Earth's triaxiality would produce a system of instantaneous moments and products of inertia with the magnitude of the equatorial product of inertia of the same order as that of the larger longitudinal product of inertia, whereas the magnitude of the latter depends upon the deviation of the two axes. In order to conserve its angular momentum or to keep its rotational energy a minimum, a deformable Earth has always a tendency to eliminate the deviation between the rotation axis and the axis of maximum principal moment of inertia (or the figure axis) through the redistribution of mass or deformation in the upper layers. Since the Earth's rotation axis is nearly fixed in space, the deformation of the upper layers implies a slow shift of the geoidal axis toward the rotation axis, with the figure axis dragged along. This is primarily the instability of the Earth's rotation axis discussed by Gold (1955); whereas the deformation or mass-redistribution effort of the fractured and inhomogeneous upper layers drives continental drift and sea-floor spreading, with the heat-generation in the interior as the energy supply. However, one should note here that for a triaxial Earth the figure axis is not really the axis of maximum principal moment of inertia but rather the axis of maximum instantaneous moment of inertia. For simplicity we may call the former the equilibrium figure axis and the latter the 
instantaneous figure axis. The present rotation ax is is rather closer to the latter while the geoidal axis is closer to the former. The four axes would eventually coincide with each other if there were no episodic energy perturbation in the Earth.

\section{The Episodic Energy Perturbation}

The episodic nature of the Earth's orogenic movements has long been recognized by geologists, which served them for the division of geological time into epochs. Recent tectonic studies (Le Pichon, 1968; Isacks et al., 1968) also find clear indications of the episodic phenomenon in the Earth's evolution history, and also in polar wandering (Irving and Robertson, 1969). Pan (1963, 1968, 1970) suggests that the episodic phenomenon could be a reflection of the energy budget or status of heat-generation in the Earth's interior. According to Pan's (1968) analysis, the energy capacity of the Earth's upper layers is about $10^{35}$ ergs, which could be reached by the excess heatgeneration in the interior in a geologic epoch, or about $10^{7} \mathrm{yr}$. With the low thermalconductive lithosphere as an insulating outer wall, the energy would mostly concentrate in the upper asthenosphere. As the energy accumulation approaches its capacity, the upper asthenosphere would start to melt while the lithosphere would overcome its critical strength. The energy accumulation would eventually provoke drastic changes in physical conditions in some more susceptible zones in the layers as forms of energy release, probably initiated by a gigantic failure of the zones. But the energy accumulation in the interior is continuous, therefore the episodic diastrophism would repeatedly occur until the exhaustion of the excess energy. This is the hypothesis of episodic energy perturbation.

\section{The Excitation Dynamics}

Pan (1968) has discussed some aspects of polar excitation by the proposed episodic gigantic failure of some susceptible zones in the upper layers, under the assumption that its mechanism was basically similar to that of earthquakes but much larger in scale because of its tremendous energy supply. The excitation function of the failure is dependent upon the assumptions concerning the scale, location and direction of the failure. However, an estimate of the excitation due to the relative motion of the failure is not difficult to make. For instance, we may assume that the rupture velocity of the failure is of the same as that of earthquakes; i.e., of the order of the shear velocity or of the rotation velocity at the equator. Then, as the failure occurs suddenly in the form of a step function, its residual acceleration would be of the same order as the velocity. This is similar to what Press (1965) called the zero-frequency seismology. If the above assumption is true, then a sudden motion of a mass of the order of $10^{16}$ to $10^{18} \mathrm{~g}$ is sufficient to produce an excitation of the order of $10^{-5}$ to $10^{-3}$. This estimate is reasonable as comparable to the mass that can be moved by a large earthquake.

The polar excitation of the gigantic failure would separate not only the rotation pole from the instantaneous figure pole, but also the instantaneous figure pole from 
the equilibrium figure pole, for near the end of an eposide the poles would be closely approaching to each other owing to the adjustment of the geoidal pole. The deviation of the two figure poles produces instantaneous products of inertia that are the continued excitation of the polar instability. According to the present analysis, the $y$ component is the major excitation and is of about the same order of magnitude as the initial separation, whereas the $x$-component is about $10^{-5}$ to $10^{-7}$, depending upon the significance of the mass redistribution of the failure. The $z$-component is much smaller, of the order of $10^{-10}$ or so. On the other hand, the damping factor of the initial wobble obtained from this analysis is quite small, of the order of $10^{-12} \mathrm{rad} / \mathrm{s}$, which implies a relaxation time of tens of thousands years to hundreds of thousands years. Furthermore, the analysis also shows that the Earth's rotation speed has not decreased linearly during geological time as is usually believed, but rather increased a certain amount (proportional to the excitation function) at the beginning of each episode and then gradually decreased with the slow damping of the wobble.

The above analysis implies that the Chandler wobble could be a quasi-permanent phenomenon in the Earth and has two components. This is in agreement with the recent observations (Gaposchkin, 1972). The incipient locus of the wobble would be elliptic, with its semi-major axis towards the direction of the secular motion or that of the axis of the intermediate instantaneous moment of inertia. However, the length of the semi-major axis would gradually decrease as the wobble slowly damped down.

\section{The Mechanisms of Wandering}

The mechanisms of polar wandering of a triaxial Earth are, as has been previously pointed out, somewhat more complex than that of the biaxial model discussed by Gold (1955), for there is an additional distinction between the instantaneous figure pole and the equilibrium figure pole in this case. That is, there is a deviation of the axes of instantaneous moments of inertia from the axes of principal moments of inertia or the principal axes of the Earth; this deviation produces the instantaneous products of inertia. Therefore, polar wandering of a triaxial Earth is a mechanism of rotational imbalances or the attempt of a disturbed Earth to seek rotational stability.

The rate of polar wandering, according to the present analysis, is of the order of $10^{-8}$ to $10^{-9} \mathrm{rad} / \mathrm{yr}$, in agreement with the observed value. With this value, the rate of change of the moments and products of inertia or the redistribution of angular momentum due to polar wandering ranges from $10^{30}$ to $10^{33} \mathrm{~g} \mathrm{~cm}^{2} / \mathrm{yr}$; the largest rate of change is that of the larger longitudinal product of inertia. The rate of change of the equatorial product of inertia is one of the smallest, about $10^{30} \mathrm{~g} \mathrm{~cm}^{2} / \mathrm{yr}$. This difference in rate of change of the products of inertia might imply a smaller rate of continental drift and sea-floor spreading than that of polar wandering. When the Earth's two equatorial moments of inertia were nearly equal and the instantaneous equatorial product of inertia negligible, rotational deformation would be the main adjustment of the Earth to polar wandering. But as the proto-continents continued to appear in one hemisphere, the instantaneous equatorial product of inertia increased 
with the difference between the two equatorial moments of inertia. Eventually the proto-continents broke up as an effect of the Earth's attempt to eliminate its products of inertia for rotational equilibrium. This mechanism may explain the late-occurrence of the major continental drift or the open-up of the Atlantic. For instance, if we assume that at the time of break-up the product of inertia of the continent-ocean system was of the same order as it is now, then the instantaneous equatorial product of inertia at that time would be at least twice greater than the present value.

Tectonically, polar wandering, continental drift and sea-floor spreading could all be thought as the mechanical reflections of the episodic energy perturbation. The incipient gigantic failure of the perturbation would continue in the form of orogenesis, whereas the tectonic layers would adjust themselves to the polar instability with epeirogenesis and continental drift. In the meantime, the torsion of the incipient wobble would fracture the lithosphere to initiate sea-floor spreading and transcurrent faulting (Pan, 1963, 1968, 1970; Rance, 1967).

\section{Conclusions}

The present analysis suggests a dynamical interpretation of the Earth's evolution, under the assumption that the Earth is a triaxial (near-symmetric), deformable and rotating body. The Earth's energy is self-generating, and the episodic release or perturbation of its excess energy furnishes dynamics for the evolution. Polar wandering is a mechanical response of a rotating Earth to the energy disequilibrium in the interior, while continental drift, sea-floor spreading and other tectonic movements in the Earth's upper layers reflect the adjustments of a triaxial deformable Earth to its polar instability. Therefore, polar wandering and tectonic movements are closely linked in their driving mechanisms; i.e., the episodic energy perturbation excites polar wandering, polar wandering maintains tectonic movements, and tectonic movements adjust polar wandering. The rate and continuation of polar wandering and tectonic movements echo the energy budget and physical conditions in the Earth's interior, whereas the direction and path of polar wandering depends rather upon the perturbation situation and the triaxiality of an episode. As long as the energy-generation in the interior is sufficient to maintain its dynamical evolution, the Earth would never be able to reach its polar stability or rotational equilibrium.

\section{Acknowledgement}

Thanks are expressed for Lady B. S. Jeffreys' critical edition of the paper.

\section{References}

Burgers, J.: 1955, Nederland. Akad. Wetensch. Proc. 58, 219.

Chinnery, M. A. and Wells, F. J.: 1972, this volume, p. 215.

Dahlen, F. A.: 1971, AGU Trans. 52, 280.

Gaposchkin, E. M.: 1972, this volume, p. 19. 
Gold, T.: 1955, Nature 175, 526.

Goldreich, P. and Toomre, A.: 1969, J. Geophys. Res. 74, 2555.

Gutenburg, B.: 1959, Physics of the Earth's Interior, Acad. Press, New York, 240 pp.

Inglis, D.: 1957, Rev. Mod. Phys. 29, 9.

Irving, E. and Robertson, W. A.: 1969, J. Geophys. Res. 74, 1026.

Isacks, B., Oliver, J., and Sykes, L. R.: 1968, J. Geophys. Res. 73, 5855.

Kaula, W. M.: 1967, Space Sci. Rev. 7, 769.

Kaula, W. M.: 1969a, J. Geophys. Res. 74, 2568.

Kaula, W. M.: 1969b, J. Geophys. Res. 74, 4807.

Kaula, W. M.: 1970, Science 169, 982.

Le Pichon, X.: 1968, J. Geophys. Res. 73, 3661.

Liu, C. P.: 1969a, $A G U$ Trans. 50, 121.

Liu, C. P.: 1969b, AGU Trans. 50, 601.

Ma, T. Y. H.: 1960, Res. Past. Clim. Cont. Drift 15, 24 pp.

Mansinha, L. and Smylie, D. E.: 1967, J. Geophys. Res. 72, 4731.

Mansinha, L. and Smylie, D. E.: 1968, Science 161, 1127.

Markowitz, W.: 1968, in Wm. Markowitz and B. Guinot (eds.), 'Continental Drift, Secular Motion of the Pole, and Rotation of the Earth', IAU Symp. 32, 25.

Munk, W. H.: 1956, Nature 177, 551.

Munk, W. H. and MacDonald, G. J. F.: 1960, The Rotation of the Earth, Cambridge Univ. Press, London, $324 \mathrm{pp}$.

Pan, C.: 1963, 'A Preliminary Study on the Relations between Polar Wandering and Seismicity',

S. M. Thesis, Dept. of Geology and Geophysics, Mass. Institute of Technology.

Pan, C.: 1968, Tectonophys. 5, 125.

Pan, C.: 1970, AGU Trans. 51, 430.

Pan, C.: 1971a, AGU Trans. 52, 355.

Pan, C.: 1971b, 'Remarks on the Excitation of the Chandler Wobble by Earthquakes', in preparation.

Press, F.: 1965, J. Geophys. Res. 70, 2395.

Rance, H.: 1967, J. Geophys. Res. 72, 2213.

Shimazaki, K. and Takeuchi, H.: 1972, this volume, p. 224.

Stoyko, A.: 1968, in Wm. Markowitz and B. Guinot (eds.), 'Continental Drift, Secular Motion of the Pole, and Rotation of the Earth', IAU Symp. 32, 52.

Takeuchi, H. and Sugi, N.: 1972, this volume, p. 212.

Thomson, W. T.: 1963, Introduction to Space Dynamics, John Wiley \& Sons, New York, 317 pp. Yumi, S. and Wako, Y.: 1968, in Wm. Markowitz and B. Guinot (eds.), 'Continental Drift, Secular Motion of the Pole, and Rotation of the Earth', IAU Symp. 32, 33.

\section{DISCUSSION}

P. Melchior: I do not understand why you propose a rheological model with many springs in parallel and only one dashpot in series. In any case it cannot represent a fracture effect. To do that you should introduce a Saint Venant element as for example in a Benioff Body. I do not see how your rheological model is introduced in the equations you have written.

C. Pan: The rheological model proposed in the presentation abstract is not final. Actually the mathematical and numerical analysis of the model has not yet been complete and is subject to future modification. The main reason to introduce a rheological model into this analysis is for the calculation of the energy dissipation in the Earth's upper layers. Therefore the rheological model will be connected to the equations in this analysis through the rates of energy dissipation of polar wandering and tectonic movements. 